\title{
Informational Energy and Its Application in Testing Normality
}

\author{
Hadi Alizadeh Noughabi ${ }^{1}$ • Majid Chahkandi ${ }^{1}$
}

Received: 25 June 2015 / Revised: 5 November 2015 / Accepted: 23 November 2015 /

Published online: 14 December 2015

(C) Springer-Verlag Berlin Heidelberg 2015

\begin{abstract}
In this article, we propose a test of fit for normality based on the estimated Informational Energy and using m-step spacings. Consistency of the test statistic is established. Critical values and power values of the test against various alternatives are calculated. Finally, the power values of the proposed test are compared with the power values of some prominent normality tests.
\end{abstract}

Keywords Informational energy · Test of normality · Test power ·

Monte Carlo simulation

\section{Introduction}

Suppose that the random variable $X$ has distribution function $F$ with density function $f$. The informational energy $\varepsilon(f)$ of the random variable was defined by

$$
\varepsilon(f)=\int_{-\infty}^{\infty} f^{2}(x) d x .
$$

Onicescu [4] justified the name informational energy and its connection to Information Theory in the classical mechanics. Rao [8] obtained distributions describing equilibrium states in statistical mechanics based on informational energy. The informational energy has been widely used in many statistical problems, see $[5,6,9]$ and references therein.

$凶$ Hadi Alizadeh Noughabi

alizadehhadi@birjand.ac.ir

1 Department of Statistics, University of Birjand, Birjand, Iran 
Pardo [7], for one-dimensional distributions, proposed an estimator of informational energy. His estimate was based on the fact that (1) can be expressed as

$$
\varepsilon(f)=\int_{0}^{1}\left(\frac{d}{d p} F^{-1}(p)\right)^{-1} d p .
$$

The estimate was constructed by replacing the distribution function $F$ by the empirical distribution function $F_{n}$, and using a difference operator instead of the differential operator. The derivative of $F^{-1}(p)$ is then estimated by a function of the order statistics. Assuming that $X_{1}, \ldots, X_{n}$ is the sample, then the estimator is given by

$$
\varepsilon_{m n}=\frac{1}{n} \sum_{i=1}^{n} \frac{2 m}{n\left(X_{(i+m)}-X_{(i-m)}\right)},
$$

where $m$ is positive integer, $m \leq \frac{n}{2}$, and $X_{(1)} \leq X_{(2)} \leq \cdots \leq X_{(n)}$ are the order statistics and $X_{(i)}=X_{(1)}$ if $i<1, X_{(i)}=X_{(n)}$ if $i>n$.

Pardo [7] showed that $\varepsilon_{m n}$ is a consistent estimator to the informational energy of $U(0,1)$ samples and it is greater or equal than one. Note that the informational energy of a $U(0,1)$ distribution is one.

In Reliability studies, engineering and management sciences, testing whether the underlying distribution has a particular form is very important and statistical methods assume an underlying distribution in the derivation of their results. Since mis-specifying the distribution may prove very costly, this problem must check carefully.

A theorem of [7] states that among all distributions that possess a density function $f$ and have a support $(0,1)$, the entropy $\varepsilon(f)$ is minimized by the uniform distribution. Based on this property, [7] introduced the following statistic for test of uniformity.

$$
\varepsilon_{m n}=\frac{1}{n} \sum_{i=1}^{n} \frac{2 m}{n\left(X_{(i+m)}-X_{(i-m)}\right)} .
$$

Large values of $\varepsilon_{m n}$ indicate that the sample is from a non-uniform distribution. Next, he obtained the percentage points of the test statistic and the power of test by simulation.

In Sect. 2, we introduce a test for normality based on informational energy. Consistency and location-scale invariance of the proposed test is established. In Sect. 3, we compare the power of the proposed test with the some prominent existing tests on a wide variety of alternatives and for sample sizes $n=10,20,30$ and 50, and show that, for some types of alternatives, the proposed test achieve higher power than the competitors.

\section{The Test Statistic}

Given a random sample $X_{1}, \ldots, X_{n}$ from a continuous probability distribution $F$ with a density $f(x)$, the hypothesis of interest is 
$H_{0}: f(x)=f_{0}(x ; \mu, \sigma)=\frac{1}{\sqrt{2 \pi} \sigma} \exp \left\{-\frac{1}{2}\left(\frac{x-\mu}{\sigma}\right)^{2}\right\}$, for some $(\mu, \sigma) \in \Theta$

where $\mu$ and $\sigma$ are unspecified and $\Theta=\mathbb{R} \times \mathbb{R}^{+}$. The alternative to $H_{0}$ is

$$
H_{1}: f(x) \neq f_{0}(x ; \mu, \sigma) \quad \text { for any }(\mu, \sigma) \in \Theta
$$

Without loss of any generality, one can reduce the above problem of goodness-of-fit, to testing the hypothesis of uniformity on the unit interval, by means of the probability integral transformation $U=F_{0}(X)$. Therefore, if $U_{i}=F_{0}\left(X_{i}\right), i=1,2, \ldots, n$ be the transformed sample, then the hypotheses can be rewrite as

$$
H_{0}: f(u)=1, \quad 0<u<1,
$$

against

$$
H_{1}: f(u) \neq 1, \quad 0<u<1 .
$$

Now, we use the test introduced by [7] for uniformity. Thus, the proposed test statistic is

$$
T_{m n}=\frac{1}{n} \sum_{i=1}^{n} \frac{2 m}{n\left(U_{(i+m)}-U_{(i-m)}\right)}=\frac{1}{n} \sum_{i=1}^{n} \frac{2 m}{n\left(F_{0}\left(X_{(i+m)}, \hat{\theta}\right)-F_{0}\left(X_{(i-m)}, \hat{\theta}\right)\right)},
$$

where $F_{0}$ is normal distribution function, $m$ is positive integer, $m \leq \frac{n}{2}, X_{(1)} \leq X_{(2)} \leq$ $\cdots \leq X_{(n)}$ are order statistics and $X_{(i)}=X_{(1)}$ if $i<1, X_{(i)}=X_{(n)}$ if $i>n$. Also $\hat{\theta}=(\hat{\mu}, \hat{\sigma})$ where

$$
\hat{\mu}=\bar{X}=\frac{1}{n} \sum_{i=1}^{n} X_{i} ; \quad \hat{\sigma}=s=\sqrt{\frac{1}{n} \sum_{i=1}^{n}\left(X_{i}-\bar{X}\right)^{2}} .
$$

It is clear that the test statistic is invariant with respect to location and scale transformations.

Remark 1 When the parameters of the distribution are specified as $\underline{\theta}=\underline{\theta}_{0}$, (that is when the null hypothesis is simple) the test statistic is

$$
T_{m n}=\frac{1}{n} \sum_{i=1}^{n} \frac{2 m}{n\left(F_{0}\left(X_{(i+m)}, \underline{\theta}_{0}\right)-F_{0}\left(X_{(i-m)}, \underline{\theta}_{0}\right)\right)} .
$$

Then, under $H_{0}$, the distribution of $T_{m n}$ is independent of $F_{0}$.

Remark 2 When the null hypothesis is composite, if $\hat{\theta} \rightarrow \theta_{0}$ as $n \rightarrow \infty$, the distribution of $T_{m n}$ at $\theta=\theta_{0}$ tends to the distribution of $T_{m n}$ under simple hypothesis. 
Similar to the argument in [7], the following theorem is stated and proved.

Theorem Let $X_{1}, \ldots, X_{n}$ be a random sample from normal distribution, we have $T_{m n} \geq 1$, and if $m=o(n)$ and $m \neq 1$, then

$$
T_{m n} \stackrel{\operatorname{Pr}}{\rightarrow} 1 \text { as } n \rightarrow \infty, m \rightarrow \infty .
$$

Proof We know that the geometric mean does not exceed from the arithmetic mean, then

$$
\begin{aligned}
T_{m n} & =\frac{1}{n} \sum_{i=1}^{n} \frac{2 m}{n\left(F_{0}\left(X_{(i+m)}, \hat{\theta}\right)-F_{0}\left(X_{(i-m)}, \hat{\theta}\right)\right)} \\
& \geq \prod_{i=1}^{n}\left(\frac{2 m}{n\left(F_{0}\left(X_{(i+m)}, \hat{\theta}\right)-F_{0}\left(X_{(i-m)}, \hat{\theta}\right)\right)}\right)^{1 / n} \\
& =\exp \left\{\frac{1}{n} \sum_{i=1}^{n} \ln \left(\frac{2 m}{n\left(F_{0}\left(X_{(i+m)}, \hat{\theta}\right)-F_{0}\left(X_{(i-m)}, \hat{\theta}\right)\right)}\right)\right\} .
\end{aligned}
$$

In other hand, we have

$$
\begin{aligned}
& \exp \left\{\frac{1}{n} \sum_{i=1}^{n} \ln \left(\frac{n\left(F_{0}\left(X_{(i+m)}, \hat{\theta}\right)-F_{0}\left(X_{(i-m)}, \hat{\theta}\right)\right)}{2 m}\right)\right\} \\
& =\prod_{i=1}^{n}\left(\frac{n\left(F_{0}\left(X_{(i+m)}, \hat{\theta}\right)-F_{0}\left(X_{(i-m)}, \hat{\theta}\right)\right)}{2 m}\right)^{1 / n} \\
& \leq \sum_{i=1}^{n} \frac{F_{0}\left(X_{(i+m)}, \hat{\theta}\right)-F_{0}\left(X_{(i-m)}, \hat{\theta}\right)}{2 m} \leq F_{0}\left(X_{(n)}, \hat{\theta}\right)-F_{0}\left(X_{(1)}, \hat{\theta}\right) \leq 1 .
\end{aligned}
$$

Therefore

$$
T_{m n} \geq \exp \left\{\frac{1}{n} \sum_{i=1}^{n} \ln \left(\frac{2 m}{n\left(F_{0}\left(X_{(i+m)}, \hat{\theta}\right)-F_{0}\left(X_{(i-m)}, \hat{\theta}\right)\right)}\right)\right\} \geq 1
$$

We know that $Y=F_{0}\left(X_{(i+j)}, \hat{\theta}\right)-F_{0}\left(X_{(i)}, \hat{\theta}\right)$ has a Beta distribution with parameters $j$ and $n-j+1$. Moreover,

$$
E\left(\frac{1}{Y}\right)=\frac{n}{j-1}
$$


Now, we calculate $E\left(T_{m n}\right)$.

$$
\begin{aligned}
E\left(T_{m n}\right)= & \frac{2 m}{n^{2}}\left\{\sum_{i=1}^{m} E\left(\frac{1}{F_{0}\left(X_{(i+m)}, \hat{\theta}\right)-F_{0}\left(X_{(1)}, \hat{\theta}\right)}\right)\right. \\
& +\sum_{i=m+1}^{n-m} E\left(\frac{1}{F_{0}\left(X_{(i+m)}, \hat{\theta}\right)-F_{0}\left(X_{(i-m)}, \hat{\theta}\right)}\right) \\
& +\sum_{i=n-m+1}^{n} E\left(\frac{1}{F_{0}\left(X_{(n)}, \hat{\theta}\right)-F_{0}\left(X_{(i-m)}, \hat{\theta}\right)}\right) \\
= & \frac{2 m}{n}\left\{\sum_{i=1}^{m} \frac{1}{i+m-2}+\frac{n-2 m}{2 m-1}+\sum_{i=n-m+1}^{n} \frac{1}{n-i+m-1}\right\} \\
= & \frac{2 m}{n}\left\{2 \sum_{i=1}^{m} \frac{1}{2 m-i-1}+\frac{n-2 m}{2 m-1}\right\} .
\end{aligned}
$$

Note that

$$
\sum_{i=1}^{m} \frac{1}{(2 m-1)-i}=\psi(2 m-1)-\psi(m-1)
$$

where $\psi$ is the digamma function. We then obtain

$$
E\left(T_{m n}\right)=\frac{2 m}{n}\left\{2 \psi(2 m-1)-2 \psi(m-1)+\frac{n-2 m}{2 m-1}\right\} .
$$

For large value of $x$, we have

$$
\psi(x) \sim \log x-\frac{1}{2 x}
$$

then when $n \rightarrow \infty, m \rightarrow \infty, m=o(n)$ and $m \neq 1$, $\lim E\left(T_{m n}\right)=\lim \left\{\frac{4 m}{n} \log \frac{2 m-1}{m-1}+\frac{2 m}{n(m-1)}+\frac{2 m}{2 m-1}-\frac{2 m(2 m+1)}{(2 m-1) n}\right\}=1$.

Therefore

$$
T_{m n} \stackrel{\text { Pr. }}{\rightarrow} 1 \text { as } n \rightarrow \infty, \quad m \rightarrow \infty .
$$




\section{Simulation Study}

We compare the power values of the proposed test with the power values of the tests which are commonly used in practice. These tests are the Cramer-von Mises $W^{2}$, the Watson $U^{2}$, the Anderson-Darling $A^{2}$, the Kolmogorov-Smirnov $D$, the Kuiper $V$ and the Shapiro-Wilk $S W$. The procedures of these tests are as follows.

Suppose $x_{(1)}, x_{(2)}, \ldots, x_{(n)}$ are the observed order statistics of the sample.

1. Find the maximum likelihood estimates of the parameters, denoted by $\hat{\theta}$.

2. Make the transformation $z_{(i)}=F_{0}\left(x_{(i)}, \hat{\theta}\right)$, for $i=1,2, \ldots, n$, where $F_{0}$ is the normal distribution function.

3. The Cramer-von Mises statistic is

$$
W^{2}=\frac{1}{12 n}+\sum_{i=1}^{n}\left(\frac{2 i-1}{2 n}-Z_{(i)}\right)^{2}
$$

The Watson statistic is computed from

$$
U^{2}=W^{2}-n\left(\bar{z}-\frac{1}{2}\right)^{2},
$$

where $\bar{z}$ is the mean of $z_{i}$, and the Anderson-Darling statistic is

$$
A^{2}=-n-\frac{1}{n} \sum_{i=1}^{n}(2 i-1)\left\{\log \left(z_{(i)}\right)+\log \left(1-z_{(n-i+1)}\right)\right\} \text {. }
$$

The Kolmogorov statistics are computed from

$$
D^{+}=\max _{1 \leq i \leq n}\left\{\frac{i}{n}-z_{(i)}\right\} ; \quad D^{-}=\max _{1 \leq i \leq n}\left\{z_{(i)}-\frac{i-1}{n}\right\},
$$

then the Kolmogorov-Smirnov statistic is $D=\max \left(D^{+}, D^{-}\right)$and the Kuiper statistic is $V=D^{+}+D^{-}$.

4. Find the percentage point $C^{*}$ at a given significance level, for sample size $n$. We can find the percentage points of these tests statistics in statistical literature. If the value of the test statistic is greater than $C^{*}$, the null hypothesis is rejected at level $\alpha$.

For small to moderate sample sizes, the critical values of the proposed test statistic are calculated by Monte Carlo simulation. Table 1 gives the critical values of $T_{m n}$ statistic for various sample sizes.

The power values of the tests based on $C H, U^{2}, D, V, A^{2}, S W$ and $T_{m n}$ statistics by means of Monte Carlo simulations under 20 alternatives are computed. These alternatives were used by [1,3] and [2] in their study of power comparisons of several tests for normality. The alternatives, depending on the support and shape of their densities, can be divided into four groups. It is clear that the natural alternatives to 
Table 1 Critical values of the $T_{m n}$ statistic at significance level $\alpha=0.05$

\begin{tabular}{|c|c|c|c|c|c|c|c|c|c|c|}
\hline \multirow[t]{2}{*}{$n$} & \multicolumn{10}{|l|}{$m$} \\
\hline & 1 & 2 & 3 & 4 & 5 & 6 & 7 & 8 & 9 & 10 \\
\hline 5 & 13.17 & 3.183 & & & & & & & & \\
\hline 6 & 11.83 & 3.135 & 2.264 & & & & & & & \\
\hline 7 & 10.78 & 3.070 & 2.194 & & & & & & & \\
\hline 8 & 10.22 & 2.997 & 2.178 & 1.973 & & & & & & \\
\hline 9 & 9.001 & 2.878 & 2.144 & 1.916 & & & & & & \\
\hline 10 & 8.603 & 2.837 & 2.121 & 1.869 & 1.829 & & & & & \\
\hline 15 & 6.608 & 2.426 & 1.941 & 1.762 & 1.669 & 1.637 & 1.640 & & & \\
\hline 20 & 5.737 & 2.187 & 1.772 & 1.656 & 1.593 & 1.555 & 1.537 & 1.532 & 1.558 & 1.591 \\
\hline 25 & 5.283 & 2.043 & 1.682 & 1.568 & 1.527 & 1.502 & 1.487 & 1.476 & 1.476 & 1.485 \\
\hline 30 & 4.752 & 1.950 & 1.615 & 1.511 & 1.462 & 1.448 & 1.436 & 1.438 & 1.433 & 1.434 \\
\hline 40 & 4.169 & 1.820 & 1.525 & 1.431 & 1.391 & 1.372 & 1.365 & 1.361 & 1.366 & 1.369 \\
\hline 50 & 4.004 & 1.744 & 1.475 & 1.383 & 1.336 & 1.321 & 1.316 & 1.311 & 1.315 & 1.316 \\
\hline
\end{tabular}

Table 2 Power comparisons of 0.05 tests based on $W^{2}, U^{2}, A^{2}, D, V, S W$ and $T_{m n}$ statistics for sample sizes $n=10,20$ under alternatives from group I

\begin{tabular}{lllllllll}
\hline$n$ & Alternatives & $W^{2}$ & $U^{2}$ & $A^{2}$ & $D$ & $V$ & $S W$ & $T_{m n}$ \\
\hline 10 & $t_{(1)}$ & $\mathbf{0 . 6 1 2}$ & 0.606 & 0.609 & 0.579 & 0.589 & 0.594 & 0.450 \\
20 & $t_{(1)}$ & 0.877 & 0.876 & $\mathbf{0 . 8 7 9}$ & 0.848 & 0.863 & 0.869 & 0.652 \\
30 & $t_{(1)}$ & 0.962 & 0.963 & $\mathbf{0 . 9 6 4}$ & 0.943 & 0.955 & 0.960 & 0.875 \\
50 & $t_{(1)}$ & 0.997 & 0.998 & $\mathbf{0 . 9 9 7}$ & 0.994 & 0.997 & $\mathbf{0 . 9 9 7}$ & 0.989 \\
10 & $t_{(3)}$ & 0.175 & 0.168 & 0.182 & 0.159 & 0.158 & $\mathbf{0 . 1 8 7}$ & 0.124 \\
20 & $t_{(3)}$ & 0.308 & 0.300 & 0.330 & 0.266 & 0.276 & $\mathbf{0 . 3 4 0}$ & 0.158 \\
30 & $t_{(3)}$ & 0.408 & 0.403 & 0.438 & 0.343 & 0.375 & $\mathbf{0 . 4 6 0}$ & 0.272 \\
50 & $t_{(3)}$ & 0.573 & 0.575 & 0.607 & 0.482 & 0.540 & $\mathbf{0 . 6 3 2}$ & 0.423 \\
10 & Logistic & 0.077 & 0.074 & 0.079 & 0.074 & 0.071 & $\mathbf{0 . 0 8 2}$ & 0.057 \\
20 & Logistic & 0.096 & 0.091 & 0.104 & 0.084 & 0.087 & $\mathbf{0 . 1 2 3}$ & 0.058 \\
30 & Logistic & 0.108 & 0.104 & 0.123 & 0.094 & 0.099 & $\mathbf{0 . 1 4 4}$ & 0.086 \\
50 & Logistic & 0.143 & 0.144 & 0.160 & 0.113 & 0.135 & $\mathbf{0 . 1 9 2}$ & 0.099 \\
10 & Laplace & 0.154 & 0.150 & $\mathbf{0 . 1 5 5}$ & 0.140 & 0.139 & 0.150 & 0.096 \\
20 & Laplace & 0.267 & 0.262 & $\mathbf{0 . 2 7 3}$ & 0.227 & 0.244 & 0.264 & 0.089 \\
30 & Laplace & 0.360 & 0.361 & $\mathbf{0 . 3 7 5}$ & 0.297 & 0.330 & 0.360 & 0.167 \\
50 & Laplace & 0.537 & 0.552 & $\mathbf{0 . 5 4 7}$ & 0.437 & 0.512 & 0.523 & 0.302 \\
\hline
\end{tabular}

normal distribution are in groups I and II. For the sake of completeness, groups III and IV are also considered. This fact shows additional insight to understand the behavior of the new test statistic $T_{m n}$. 
Table 3 Power comparisons of 0.05 tests based on $W^{2}, U^{2}, A^{2}, D, V, S W$ and $T_{m n}$ statistics for sample sizes $n=10,20$ under alternatives from group II

\begin{tabular}{lllllllll}
\hline$n$ & Alternatives & $W^{2}$ & $U^{2}$ & $A^{2}$ & $D$ & $V$ & $S W$ & $T_{m n}$ \\
\hline 10 & Gumbel $(0,1)$ & 0.131 & 0.123 & 0.138 & 0.116 & 0.114 & $\mathbf{0 . 1 5 3}$ & 0.129 \\
20 & Gumbel $(0,1)$ & 0.246 & 0.218 & 0.274 & 0.202 & 0.190 & $\mathbf{0 . 3 1 3}$ & 0.269 \\
30 & Gumbel $(0,1)$ & 0.349 & 0.305 & 0.392 & 0.282 & 0.267 & $\mathbf{0 . 4 6 9}$ & 0.466 \\
50 & Gumbel $(0,1)$ & 0.545 & 0.477 & 0.601 & 0.438 & 0.423 & 0.686 & $\mathbf{0 . 6 9 3}$ \\
10 & Gumbel $(0,2)$ & 0.130 & 0.124 & 0.139 & 0.115 & 0.113 & $\mathbf{0 . 1 5 0}$ & 0.135 \\
20 & Gumbel $(0,2)$ & 0.247 & 0.217 & 0.275 & 0.203 & 0.191 & $\mathbf{0 . 3 1 5}$ & 0.271 \\
30 & Gumbel $(0,2)$ & 0.350 & 0.304 & 0.391 & 0.282 & 0.266 & $\mathbf{0 . 4 6 7}$ & 0.465 \\
50 & Gumbel $(0,2)$ & 0.544 & 0.478 & 0.600 & 0.436 & 0.424 & 0.685 & $\mathbf{0 . 6 9 4}$ \\
10 & Gumbel $(0,1 / 2)$ & 0.130 & 0.125 & 0.137 & 0.117 & 0.114 & $\mathbf{0 . 1 5 4}$ & 0.130 \\
20 & Gumbel $(0,1 / 2)$ & 0.248 & 0.217 & 0.274 & 0.202 & 0.192 & $\mathbf{0 . 3 1 4}$ & 0.266 \\
30 & Gumbel $(0,1 / 2)$ & 0.351 & 0.305 & 0.393 & 0.281 & 0.268 & $\mathbf{0 . 4 6 8}$ & 0.465 \\
50 & Gumbel $(0,1 / 2)$ & 0.545 & 0.476 & 0.602 & 0.437 & 0.422 & 0.687 & $\mathbf{0 . 6 9 2}$ \\
\hline
\end{tabular}

Group I: Support $(-\infty, \infty)$, symmetric.

- Student t with 1 degree of freedom (i.e. the standard Cauchy),

- Student $t$ with 3 degrees of freedom,

- Standard logistic,

- Standard Laplace.

Group II: Support $(-\infty, \infty)$, asymmetric.

- Gumbel with parameters $\alpha$ (location) and $\beta$ (scale), denoted by $\operatorname{Gumbel}(\alpha, \beta)$

Group III: Support $(0, \infty)$.

- Exponential with mean 1 ,

- Gamma with parameter $\alpha$ (shape),

- Lognormal with parameters $\mu$ (location) and $\sigma$ (scale), denoted by Lognormal $(\mu, \sigma)$

- Weibull with parameter $\alpha$ (shape),

Group IV: Support $(0,1)$.

- Uniform,

- Beta $(2,2)$,

- Beta $(0.5,0.5)$,

- Beta $(3,1.5)$,

- Beta $(2,1)$.

Under each alternative, we generated 20,000 samples of size 10, 20, 30 and 50 and then computed the test statistics $\left(W^{2}, D, V, U^{2}, A^{2}, S W, T_{m n}\right)$. By the frequency of the event "the test statistic is in the critical region" the power value of the corresponding test was obtained. The power values are presented in Tables 2, 3, 4 and 5. For each sample size and alternative, the bold type in these Tables indicates the statistics achieving the maximum power. 
Table 4 Power comparisons of 0.05 tests based on $W^{2}, U^{2}, A^{2}, D, V, S W$ and $T_{m n}$ statistics for sample sizes $n=10,20$ under alternatives from group III

\begin{tabular}{|c|c|c|c|c|c|c|c|c|}
\hline$n$ & Alternatives & $W^{2}$ & $U^{2}$ & $A^{2}$ & $D$ & $V$ & $S W$ & $T_{m n}$ \\
\hline 10 & Exponential & 0.384 & 0.367 & 0.411 & 0.305 & 0.365 & 0.442 & 0.468 \\
\hline 20 & Exponential & 0.726 & 0.688 & 0.776 & 0.585 & 0.696 & 0.836 & 0.877 \\
\hline 30 & Exponential & 0.896 & 0.866 & 0.934 & 0.783 & 0.884 & 0.968 & 0.982 \\
\hline 50 & Exponential & 0.991 & 0.984 & 0.997 & 0.961 & 0.991 & 0.9995 & 0.9996 \\
\hline 10 & Gamma (2) & 0.203 & 0.191 & 0.217 & 0.169 & 0.181 & 0.239 & 0.237 \\
\hline 20 & Gamma (2) & 0.414 & 0.374 & 0.459 & 0.324 & 0.349 & 0.532 & 0.553 \\
\hline 30 & Gamma (2) & 0.587 & 0.530 & 0.654 & 0.466 & 0.506 & 0.749 & 0.807 \\
\hline 50 & Gamma (2) & 0.832 & 0.778 & 0.888 & 0.697 & 0.768 & 0.949 & 0.965 \\
\hline 10 & Gamma (1/2) & 0.672 & 0.658 & 0.701 & 0.541 & 0.669 & 0.735 & 0.772 \\
\hline 20 & Gamma (1/2) & 0.950 & 0.940 & 0.968 & 0.881 & 0.953 & 0.984 & 0.991 \\
\hline 30 & Gamma (1/2) & 0.996 & 0.993 & 0.998 & 0.983 & 0.997 & 0.9997 & 0.9998 \\
\hline 50 & Gamma (1/2) & 1.000 & 1.000 & 1.000 & 1.000 & 1.000 & 1.000 & 1.000 \\
\hline 10 & Lognormal $(0,1)$ & 0.552 & 0.536 & 0.576 & 0.461 & 0.527 & 0.603 & 0.626 \\
\hline 20 & Lognormal $(0,1)$ & 0.884 & 0.864 & 0.908 & 0.797 & 0.860 & 0.932 & 0.947 \\
\hline 30 & Lognormal $(0,1)$ & 0.973 & 0.962 & 0.983 & 0.934 & 0.964 & 0.991 & 0.995 \\
\hline 50 & Lognormal $(0,1)$ & 0.999 & 0.998 & 0.999 & 0.995 & 0.999 & 0.9999 & 0.9999 \\
\hline 10 & Lognormal $(0,2)$ & 0.894 & 0.889 & 0.907 & 0.824 & 0.894 & 0.920 & 0.938 \\
\hline 20 & Lognormal $(0,2)$ & 0.998 & 0.997 & 0.998 & 0.992 & 0.998 & 0.9996 & 0.9998 \\
\hline 30 & Lognormal $(0,2)$ & 1.000 & 1.000 & 1.000 & 1.000 & 1.000 & 1.000 & 1.000 \\
\hline 50 & Lognormal $(0,2)$ & 1.000 & 1.000 & 1.000 & 1.000 & 1.000 & 1.000 & 1.000 \\
\hline 10 & $\operatorname{Lognormal}(0,1 / 2)$ & 0.218 & 0.206 & 0.2231 & 0.187 & 0.192 & 0.245 & 0.230 \\
\hline 20 & Lognormal $(0,1 / 2)$ & 0.425 & 0.388 & 0.465 & 0.346 & 0.352 & 0.517 & 0.507 \\
\hline 30 & $\operatorname{Lognormal}(0,1 / 2)$ & 0.595 & 0.540 & 0.652 & 0.482 & 0.498 & 0.726 & 0.754 \\
\hline 50 & Lognormal $(0,1 / 2)$ & 0.824 & 0.769 & 0.869 & 0.706 & 0.737 & 0.924 & 0.937 \\
\hline 10 & Weibull (1/2) & 0.854 & 0.848 & 0.874 & 0.752 & 0.856 & 0.894 & 0.915 \\
\hline 20 & Weibull (1/2) & 0.995 & 0.994 & 0.997 & 0.984 & 0.996 & 0.9992 & 0.9994 \\
\hline 30 & Weibull (1/2) & 1.000 & 1.000 & 1.000 & 1.000 & 1.000 & 1.000 & 1.000 \\
\hline 50 & Weibull (1/2) & 1.000 & 1.000 & 1.000 & 1.000 & 1.000 & 1.000 & 1.000 \\
\hline 10 & Weibull (2) & 0.076 & 0.074 & 0.078 & 0.070 & 0.70 & 0.084 & 0.084 \\
\hline 20 & Weibull (2) & 0.121 & 0.110 & 0.132 & 0.105 & 0.096 & 0.156 & 0.160 \\
\hline 30 & Weibull (2) & 0.159 & 0.138 & 0.187 & 0.138 & 0.119 & 0.232 & 0.280 \\
\hline 50 & Weibull (2) & 0.261 & 0.218 & 0.308 & 0.207 & 0.182 & 0.416 & 0.489 \\
\hline
\end{tabular}

For the proposed test, the maximum power was typically attained by choosing $m=4$ for $n=10, m=7$ for $n=20, m=12$ for $n=30$, and $m=20$ for $n=50$. With increasing $n$ the optimal choice of $m$ increases.

From Tables 2, 3, 4 and 5, it is seen that the tests compared considerably differ in power. Tables 4 and 5 indicate a superiority of our procedure to other tests. In these tables, the proposed test out performs other prominent tests under more alternatives. 
Table 5 Power comparisons of 0.05 tests based on $W^{2}, U^{2}, A^{2}, D, V, S W$ and $T_{m n}$ statistics for sample sizes $n=10,20$ under alternatives from group IV

\begin{tabular}{lllllllll}
\hline$n$ & Alternatives & $W^{2}$ & $U^{2}$ & $A^{2}$ & $D$ & $V$ & $S W$ & $T_{m n}$ \\
\hline 10 & Uniform & 0.071 & 0.078 & 0.076 & 0.064 & 0.081 & 0.082 & $\mathbf{0 . 0 8 9}$ \\
20 & Uniform & 0.144 & 0.163 & 0.172 & 0.101 & 0.151 & 0.200 & $\mathbf{0 . 2 6 9}$ \\
30 & Uniform & 0.227 & 0.258 & 0.301 & 0.147 & 0.233 & $\mathbf{0 . 3 8 1}$ & 0.311 \\
50 & Uniform & 0.439 & 0.487 & 0.575 & 0.261 & 0.428 & $\mathbf{0 . 7 4 9}$ & 0.671 \\
10 & Beta(2,2) & 0.044 & 0.048 & 0.045 & 0.042 & 0.050 & 0.042 & $\mathbf{0 . 0 5 0}$ \\
20 & Beta(2,2) & 0.055 & 0.060 & 0.056 & 0.052 & 0.060 & 0.053 & $\mathbf{0 . 0 9 0}$ \\
30 & $\operatorname{Beta}(2,2)$ & 0.070 & 0.079 & 0.080 & 0.060 & 0.081 & $\mathbf{0 . 0 8 0}$ & 0.078 \\
50 & $\operatorname{Beta}(2,2)$ & 0.111 & 0.129 & 0.130 & 0.080 & 0.126 & $\mathbf{0 . 1 5 3}$ & 0.128 \\
10 & $\operatorname{Beta}(1 / 2,1 / 2)$ & 0.222 & 0.243 & 0.256 & 0.155 & 0.234 & $\mathbf{0 . 2 9 9}$ & 0.276 \\
20 & $\operatorname{Beta}(1 / 2,1 / 2)$ & 0.509 & 0.547 & 0.621 & 0.332 & 0.492 & $\mathbf{0 . 7 2 7}$ & 0.718 \\
30 & $\operatorname{Beta}(1 / 2,1 / 2)$ & 0.739 & 0.773 & 0.861 & 0.507 & 0.703 & $\mathbf{0 . 9 4 4}$ & 0.879 \\
50 & $\operatorname{Beta}(1 / 2,1 / 2)$ & 0.958 & 0.968 & 0.991 & 0.805 & 0.940 & $\mathbf{0 . 9 9 9}$ & 0.999 \\
10 & $\operatorname{Beta}(3,1 / 2)$ & 0.527 & 0.515 & 0.561 & 0.409 & 0.524 & 0.609 & $\mathbf{0 . 6 5 1}$ \\
20 & $\operatorname{Beta}(3,1 / 2)$ & 0.878 & 0.858 & 0.915 & 0.751 & 0.881 & 0.948 & $\mathbf{0 . 9 7 1}$ \\
30 & $\operatorname{Beta}(3,1 / 2)$ & 0.977 & 0.969 & 0.991 & 0.930 & 0.981 & 0.997 & $\mathbf{0 . 9 9 9}$ \\
50 & $\operatorname{Beta}(3,1 / 2)$ & 0.999 & 0.999 & 0.9999 & 0.998 & 0.9997 & 1.000 & $\mathbf{1 . 0 0 0}$ \\
10 & $\operatorname{Beta}(2,1)$ & 0.112 & 0.111 & 0.117 & 0.095 & 0.108 & 0.130 & $\mathbf{0 . 1 4 9}$ \\
20 & $\operatorname{Beta}(2,1)$ & 0.232 & 0.223 & 0.263 & 0.176 & 0.202 & 0.306 & $\mathbf{0 . 4 1 4}$ \\
30 & $\operatorname{Beta}(2,1)$ & 0.364 & 0.345 & 0.431 & 0.273 & 0.318 & 0.515 & $\mathbf{0 . 6 1 9}$ \\
50 & $\operatorname{Beta}(2,1)$ & 0.611 & 0.586 & 0.720 & 0.455 & 0.563 & 0.838 & $\mathbf{0 . 8 9 2}$ \\
\hline
\end{tabular}

The Anderson-Darling and Shapiro-Wilk tests have the most power in group I. In group II, it is observed that for small sample sizes the Shapiro-Wilk test achieves greatest power and for large sample sizes the proposed test has the most power. In the other groups, it is seen that the proposed test $T_{m n}$ has the most power. The difference of powers of the proposed test $T_{m n}$ and other tests are substantial.

\section{Conclusions}

In this paper, we first discussed about informational energy of a continuous random variable. We next proposed a new test for normality based on an estimator of informational energy. Consistency and other properties of the proposed test statistic have been shown.

The paper also compared the power vales of the proposed test with some prominent existing tests using Monte Carlo computations for sample sizes $n=10,20,30$ and 50. Differences in power values of the proposed test with other tests are considerable and each of the tests $A^{2}, S W$ and $T_{m n}$ can be most powerful depending on the type of alternatives. The tests $A^{2}$ and $S W$ are most powerful against symmetric alternatives 
with the support $(-\infty, \infty)$ (group I). The test $S W$ is most powerful against asymmetric alternatives in group II with the support $(-\infty, \infty)$.

The test $T_{m n}$ (proposed test) is most powerful against alternatives in group III with the support $(0, \infty)$ and alternatives with the support $(0,1)$ (group IV).

Based on these observations, the following recommendations for the application of the studied tests in practice are presented.

1. Use the statistics $A^{2}$ or $S W$, if the assumed alternatives are symmetric and supported by $(-\infty, \infty)$.

2. Use the statistic $S W$, if the assumed alternatives are asymmetric and supported by $(-\infty, \infty)$.

3. Use the statistic $T_{m n}$ (the proposed test), if the assumed alternatives are supported by the bounded interval $(0,1)$ or if they are supported by $(0, \infty)$.

Acknowledgments The authors are grateful to the anonymous referees and the associate editor for providing some useful comments on an earlier version of this manuscript.

\section{References}

1. Alizadeh Noughabi H (2010) A new estimator of entropy and its application in testing normality. J Stat Comput Simul 80:1151-1162

2. Alizadeh Noughabi H, Arghami NR (2011) Monte Carlo comparison of seven normality tests. J Stat Comput Simul 81:965-972

3. Esteban MD, Castellanos ME, Morales D, Vajda I (2001) Monte Carlo comparison of four normality tests using different entropy estimates. Commun Statistics-Simul Comput 30:761-785

4. Onicescu O (1966) Energie informationelle. C R Acad Sci Paris Ser A 263:841-8842

5. Onicescu O, Stefanescu V (1979) Elemente de statistica informationala eu aplicatii. Editura Technica, Bucarest

6. Pardo L, Taneja IJ (1991) Information energy and its applications. Adv Electron Electron Phys 80:165241

7. Pardo MC (2003) A test for uniformity based on informational energy. Stat Pap 44:521-534

8. Rao CR (1973) Linear statistical inference and its applications. John Wiley, New York

9. Theodorescu A (1977) Energie informationnelle et notions apparentees. Trabajos de Estadistica e I.O. 27:276-298 